\title{
Full Title: Radial artery perforation treated with balloon tracking and guide catheter tamponade - a case series
}

Short Title: Successful treatment of radial artery perforation

Authors: Sudhakar George ${ }^{1}$ MD MRCP, Mamas Mamas² DPhil MRCP, James Nolan² MD FRCP, Karim Ratib ${ }^{1}$ MRCP

${ }^{1}$ Royal Stoke University Hospital, Stoke-on-Trent, United Kingdom

${ }^{2}$ Keele Cardiovascular Research Group, Keele University, Stoke-on-Trent

Indexing words: angioplasty; complication; radial perforation; balloon assisted tracking

Address for correspondence: Sudhakar George, Department of Cardiology, Royal Stoke University Hospital, UK

Telephone +447595944223

Email: sudhakargeorge@gmail.com

Fax: +441902694063

Total word count: 2813

\section{Disclosures :}

We the authors are happy to declare that we have no conflicts of interest pertaining to any of the content in this manuscript. No extra funding was required from any organisation for the case series described. 


\begin{abstract}
A 78 year old woman was admitted with an acute coronary syndrome. She underwent coronary angiography via the left radial artery which was complicated by a guide catheter induced radial artery perforation confirmed by extravasation of contrast into the forearm. We used balloon assisted tracking and guide catheter tamponade to complete the angioplasty procedure via the radial artery and successfully seal the radial artery perforation. We describe a series of 7 cases where this technique was used to both treat radial artery perforation and to complete the angioplasty via the radial approach.
\end{abstract}

\title{
Introduction
}

The transradial access (TRA) site has been increasingly adopted as the preferred access site for percutaneous coronary intervention ( $\mathrm{PCl}$ ) across Europe and many North American Centres. Whilst transfemoral angiography and angioplasty has been the primary access site for many institutions, the number of procedures performed via the radial route has increased dramatically, particularly in Europe. One of the most important reasons for this is that TRA is associated with decreased mortality rates in high-risk patient groups, at least in part mediated through a reduction in major vascular access site related bleeding complications(1). Although very rare, access site complications may also occur in the radial artery and spasm, dissection, haematoma, haemorrhage, pseudoaneurysm formation, arterio-venous fistula formation and perforations have been reported in the literature. Radial artery perforations are more likely to occur in small or tortuous vessels and can be caused by radial sheaths, guide wires, diagnostic catheters or guiding catheters and are described in less than $0.5 \%$ of $\operatorname{cases}(2)$. In the presence of a radial perforation, successful completion of the procedure may necessitate switching to an alternative access site with its attendant risks. Furthermore, continuous haemorrhage and haematoma formation can occur as a result of radial perforation and can rarely lead to complications including compartment syndrome in the forearm requiring surgical intervention. We report a large series of cases in our high volume institution where radial artery perforations were managed using the technique of balloon assisted tracking (BAT) first described by Patel et al(3) that allowed the procedure to continue via the affected artery and resulted in successful sealing of the perforation. This technique may reduce the impact of this rare but important complication of TRA PCI.

\section{Case series}

\section{Patient A}

A 78 year old woman was admitted with an acute coronary syndrome (ACS). The patient underwent urgent coronary angiography using a 6 French ( $\mathrm{Fr}$ ) radial sheath (Terumo Corporation) in the left radial artery. Diagnostic coronary angiography was performed using 6Fr Judkins Right (JR) 4.0 and Judkins Left (JL) 3.5 catheters. This showed a critical lesion in the right coronary artery (RCA). The 
diagnostic catheter was removed over a 0.035 inch guide wire and an attempt was made to exchange to a 6Fr JR 4.0 guide catheter. The patient complained of pain over the left forearm and there was resistance to the advancing catheter. Injection of diluted contrast via the radial sheath revealed a guide catheter induced radial artery perforation with active extravasation of contrast (figure 1a). A 5Fr JR diagnostic catheter was inserted and crossed the perforation in the radial artery without difficulty. This was placed in the aortic root and the 0.035 inch guide wire $(120 \mathrm{~cm})$ removed and replaced with a 0.014 inch Sion blue coronary wire $(180 \mathrm{~cm})$. The JR diagnostic was then removed whilst the Sion blue wire remained in the aortic root.

We then used the technique of balloon assisted tracking (BAT) to attempt to introduce the $6 \mathrm{Fr}$ guiding catheter into the aortic root. To perform balloon assisted tracking, a compliant $2.0 \mathrm{~mm}$ by $15 \mathrm{~mm}$ balloon was inserted into the guiding catheter and advanced until the distal two thirds of the balloon protruded outside the tip of the catheter. The balloon was then inflated to nominal pressure. The balloon and guiding catheter were then loaded onto the back of the Sion blue wire and advanced gently across the site of the perforation (figure $1 \mathrm{~b}$ ). This manoeuvre allowed the guiding catheter to safely bypass the site of perforation and be used to engage the RCA, with successful angioplasty performed with the insertion of a single drug eluting stent. After removal of the guiding catheter, a further angiogram of the left radial artery revealed that the perforation of the radial artery had successfully been sealed (figure 1c). The following day the patient was found to have minor bruising over the forearm but no haematoma and was discharged home.

\section{Patient B}

A 63 year old man with a history of hypertension was admitted with an ACS. Following diagnostic angiography showing RCA disease, a guiding catheter induced a right radial artery perforation (figure 2a). Exchange to a $180 \mathrm{~cm} 0.018$ coronary guide wire and BAT allowed delivery of a $6 \mathrm{Fr}$ guiding catheter and successful PCI to the RCA. A radial angiogram at the end of the procedure showed the radial artery perforation had been sealed (figure $2 b$ ).

\section{Patient C}

A 66 year old man was admitted with an ACS. Diagnostic angiography via the right radial artery showed severe disease of the distal left main stem (LMS). Upon switching to a larger guiding catheter, advancement of the 6F Extra Backup (EBU) guiding catheter was met with resistance and pain. Angiography via the radial sheath revealed a small calibre axillary radial artery with perforation in the forearm (figure 3a). A 0.014 inch coronary wire was advanced into the aortic root and BAT (figure $3 \mathrm{~b}$ ) allowed delivery of the EBU catheter and PCI to the LMS was performed without difficulty. A radial angiogram at the end of the case confirmed that the perforation had been sealed (figure 3c). 


\section{Patient D}

A 51 year old man with a history of hypercholesterolaemia was admitted with an ACS. Angiography via the right radial artery showed severe disease in the RCA. A radial artery perforation was caused by a 6Fr JR 4.0 guiding catheter (figure 4a). Again, exchange to a coronary guide wire followed by the use of BAT (figure $4 \mathrm{~b}$ ) allowed deliver of the guiding catheter to the RCA and successful completion of the case. An angiogram confirmed the radial artery was again patent (figure 4c).

\section{Patient E}

An 85 year old lady with a history of hypertension was admitted with an inferior ST elevation myocardial infarction (STEMI). Diagnostic angiography via the right radial artery confirmed an occluded RCA. Advance of a JR 4.0 guiding catheter caused radial artery perforation (figure 5a). BAT allowed delivery of the guiding catheter and the RCA was stented. A final angiogram via the radial sheath showed that although the radial artery was open, there was continued extravasation of contrast, confirming that the perforation was not fully sealed (figure 5b). External compression was applied after removal of the radial sheath and the patient was discharged home 3 days later without further complication.

\section{Patient F}

A 69 year old lady with a history of diabetes mellitus and hypertension was admitted with an ACS. Diagnostic angiography via the right radial artery showed severe disease in the RCA. A radial perforation was caused by a JR 4.0 guiding catheter (figure 6a). BAT (figure 6b) allowed successful angioplasty and the perforation was sealed by the end of the procedure (figure $6 \mathrm{c}$ ).

\section{Patient G}

A 41 year old male smoker was admitted with an ACS. Diagnostic angiography showed severe disease in the LAD but advance of an EBU 3.75 guide catheter caused a large perforation (figure 7a). BAT allowed successful angioplasty and the perforation was sealed by the end of the procedure (figure 7b).

\section{Discussion}

Radial artery perforation is a rare but important complication that may occur during radial procedures, particularly in small or tortuous vessels. It frequently presents with spasm and pain and results in difficulty in passing wires and catheters. Radial artery perforation is often associated with radial artery spasm which can be lessened with the use of intra-arterial vasodilators such as verapamil and nitroglycerine(4). Difficulty is often encountered when larger Fr guiding catheters are being inserted or when there is a high degree of tortuosity. The procedure via the radial artery is often abandoned at this stage as the perforation can lead to an increase in spasm and to concern 
that the perforation can be worsened. Angiography of the arm vessels when difficulties are encountered is essential to diagnose perforation as forearm haematoma following the procedure may be the first sign of an operative complication. A delay in this important diagnosis may increase the risk of an unrecognised compartment syndrome.

Standard management of radial artery perforations previously included abandoning the procedure and applying pressure, either manually or with the use of a sphygmomanometer. This can result in stasis of blood and increase the risk of radial artery occlusion(5). As radial experience has increased, so has the range of treatment options for radial artery perforations. Groups have demonstrated that radial artery perforations can be sealed with the use of a long arterial sheath in the radial artery up to the brachial artery (6). Others have simply used the tamponading effect of a diagnostic catheter or a guiding catheter to seal radial perforations(2). Operators have even described prolonged balloon inflation at the site of the perforation and even treatment with a polytetrafluoroethylene (PTFE) covered coronary stents to seal the perforation(7). The key feature of all these techniques is that they require the presence of a wire across the perforated segment of radial artery. If radial artery perforation is caused by the radial sheath, or diagnostic catheter, the operator is unlikely to have a wire already across the perforation. In that situation, an attempt can be made at crossing the perforated segment with a 0.014 inch wire from the distal radial artery. When this is impossible (for example in a large perforation where the wire simply exits the vessel rather than entering the true lumen more proximally) some operators have described puncturing the contralateral radial artery or femoral artery to re-establish wire control over the perforation via a retrograde approach.

The advantage during our cases was that as the perforations were caused by the guiding catheter, we had already established wire position and we did not need to attempt to re-cross the perforated segment of radial artery. We were able to use BAT to safely deliver a 6Fr guiding catheter to the coronary artery and perform angioplasty. The shape of the inflated balloon at the tip of the catheter provides a tapered interface for advance of the catheter. We did not experience any difficulty in performing BAT using 6Fr guiding catheters in any of the 7 cases described. BAT has previously been described in negotiating tortuous radial arteries and radial artery spasm(3). There is also a case report of its use in a small radial perforation by the original team to perform BAT(8). Our larger case series builds on this experience and demonstrates that BAT following radial artery perforation is a useful additional technique in allowing the advance of a large guiding catheter across the perforated radial segment. This has the advantage of allowing completion of the angioplasty procedure via the radial artery and also allows the guiding catheter to act as an internal haemostatic device to seal the radial artery perforation. In 6 of our 7 patients, the radial perforation was sealed by the end of the procedure. In patient $E$, the combination of a large perforation and a relatively short procedure time meant that although BAT allowed successful angioplasty, external compression was also required at the end of the case. Our case series demonstrates that the technique can be used safely, reliably and effectively in treating radial artery perforation.

\section{Conclusion}

The use of balloon tracking and guiding catheter tamponade can be used to successfully seal radial artery perforations during $\mathrm{PCl}$ and enable successful completion of the procedure via the arm. 


\section{Figure legend}

Figure $1 \mathrm{a}$ - Perforation of the right radial artery with extravasation of contrast in Patient $A$

Figure $1 \mathrm{~b}-$ Balloon assisted tracking (BAT) of the guiding catheter in Patient A

Figure $1 c-$ Sealed perforation of the right radial artery with no evidence of contrast extravasation in Patient $\mathrm{A}$ after BAT

Figure $2 \mathrm{a}-$ Perforation of the radial artery in Patient $\mathrm{B}$

Figure $2 b-$ Sealed perforation of the radial artery in Patient B after BAT

Figure 3a- Perforation of the radial artery in Patient C

Figure $3 b-B A T$ of the guiding catheter in Patient C

Figure 3c - Sealed perforation of the radial artery in Patient C after BAT

Figure 4a- Perforation of the radial artery in Patient D

Figure $4 \mathrm{~b}$ - BAT of the guiding catheter in Patient D

Figure $4 c-$ Sealed perforation of the radial artery in Patient $D$ after BAT

Figure 5a-Radial perforation in patient $\mathrm{E}$

Figure $5 b$ - Radial artery open at end of procedure but note continued extravasation of contrast in patient E confirming that perforation not sealed

Figure 6a-Radial artery perforation in patient $\mathrm{F}$

Figure $6 b-B A T$ in patient $F$

Figure $6 c-$ Sealed radial perforation in patient $\mathrm{F}$

Figure 7a-Radial artery perforation in patient G

Figure $7 b$ - Sealed radial artery perforation after BAT in patient G 


\section{References}

1. Mamas MA, Ratib K, Routledge H, Neyses L, Fraser DG, de Belder M, et al. Influence of arterial access site selection on outcomes in primary percutaneous coronary intervention: are the results of randomized trials achievable in clinical practice? JACC Cardiovasc Interv. 2013 Jul;6(7):698-706.

2. Patel T, Shah S, Sanghavi K, Pancholy S. Management of radial and brachial artery perforations during transradial procedures--a practical approach. J Invasive Cardiol. 2009 Oct;21(10):544-7.

3. Patel T, Shah S, Pancholy S. Balloon-assisted tracking of a guide catheter through difficult radial anatomy: a technical report. Catheter Cardiovasc Interv Off J Soc Card Angiogr Interv. 2013 Apr;81(5):E215-218.

4. Kwok CS, Rashid M, Fraser D, Nolan J, Mamas M. Intra-arterial vasodilators to prevent radial artery spasm: a systematic review and pooled analysis of clinical studies. Cardiovasc Revascularization Med Mol Interv. 2015 Dec;16(8):484-90.

5. Rashid M, Kwok CS, Pancholy S, Chugh S, Kedev SA, Bernat I, et al. Radial Artery Occlusion After Transradial Interventions: A Systematic Review and Meta-Analysis. J Am Heart Assoc. 2016;5(1).

6. Calviño-Santos RA, Vázquez-Rodríguez JM, Salgado-Fernández J, Vázquez-González N, PérezFernández R, Vázquez-Rey E, et al. Management of iatrogenic radial artery perforation. Catheter Cardiovasc Interv Off J Soc Card Angiogr Interv. 2004 Jan;61(1):74-8.

7. Sallam MM, Ali M, Al-Sekaiti R. Management of radial artery perforation complicating coronary intervention: a stepwise approach. J Intervent Cardiol. 2011 Oct;24(5):401-6.

8. Deora S, Shah S, Patel T. Balloon-assisted tracking of guide catheter dealing with radial artery perforation and subclavian loop during percutaneous coronary intervention by transradial approach. Int J Cardiol. 2013 Sep 10;167(6):e161-162. 


\section{Figures}

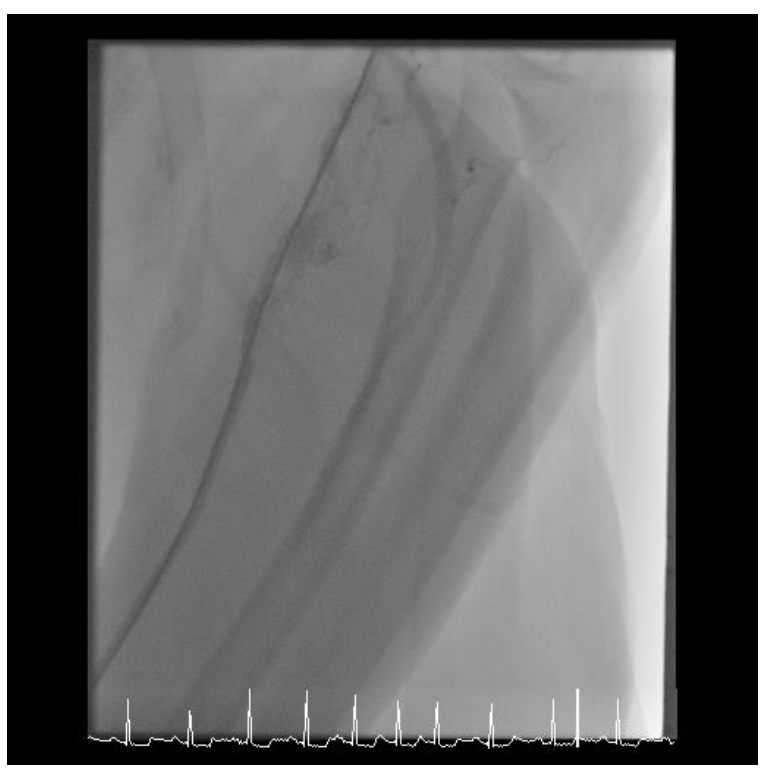

Figure 1a-Perforation of the right radial artery with extravasation of contrast in Patient A

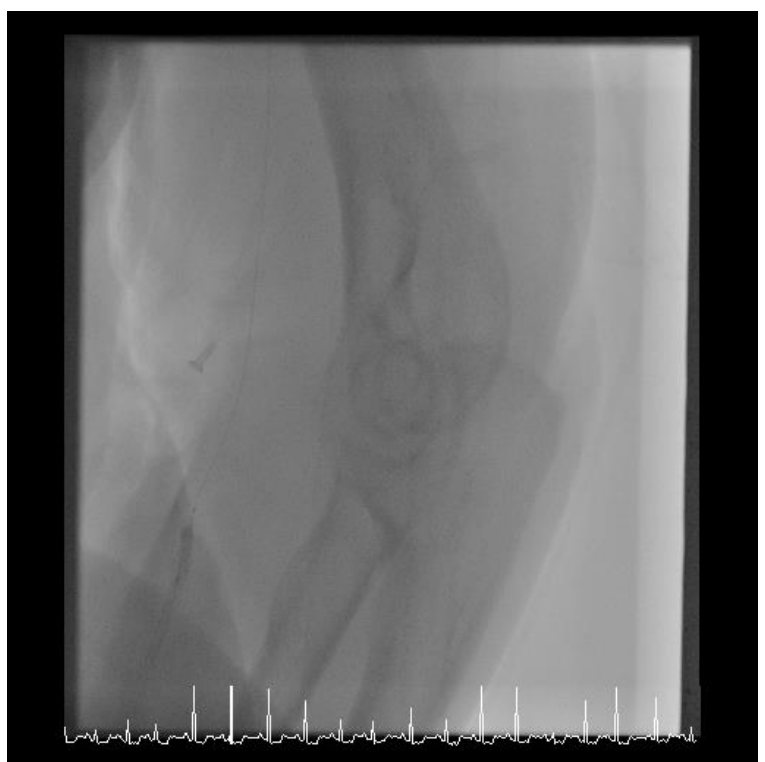

Figure 1b- Balloon assisted tracking (BAT) of the guiding catheter in Patient A 


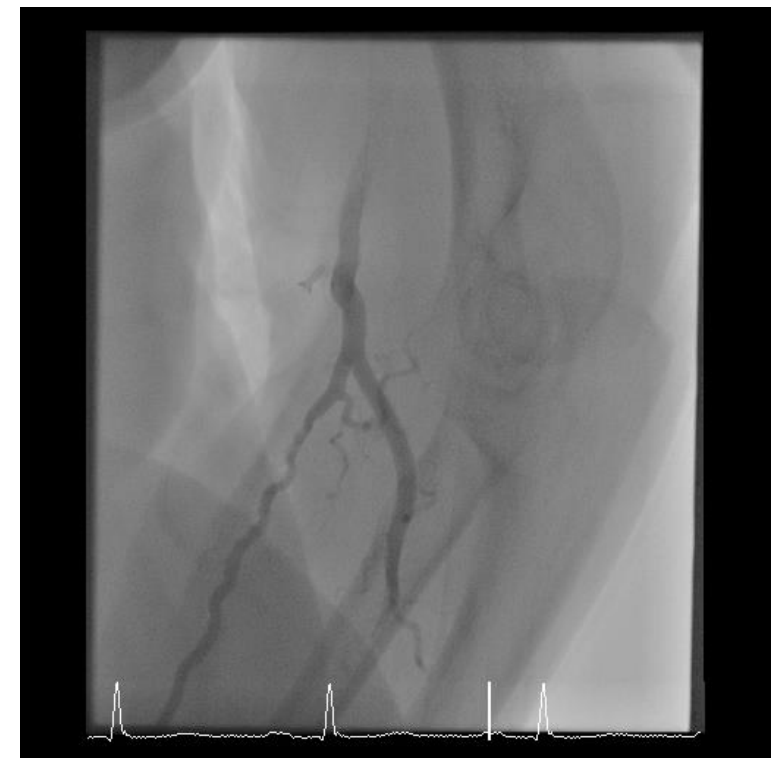

Figure 1c - Sealed perforation of the right radial artery with no evidence of contrast extravasation in Patient A after BAT

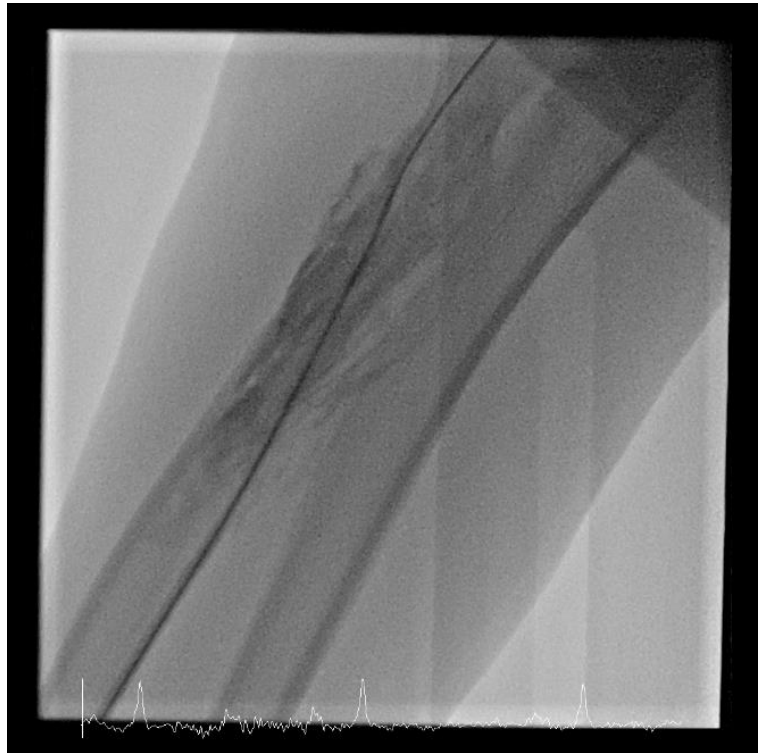

Figure $2 \mathrm{a}$ - Perforation of the radial artery in Patient B 


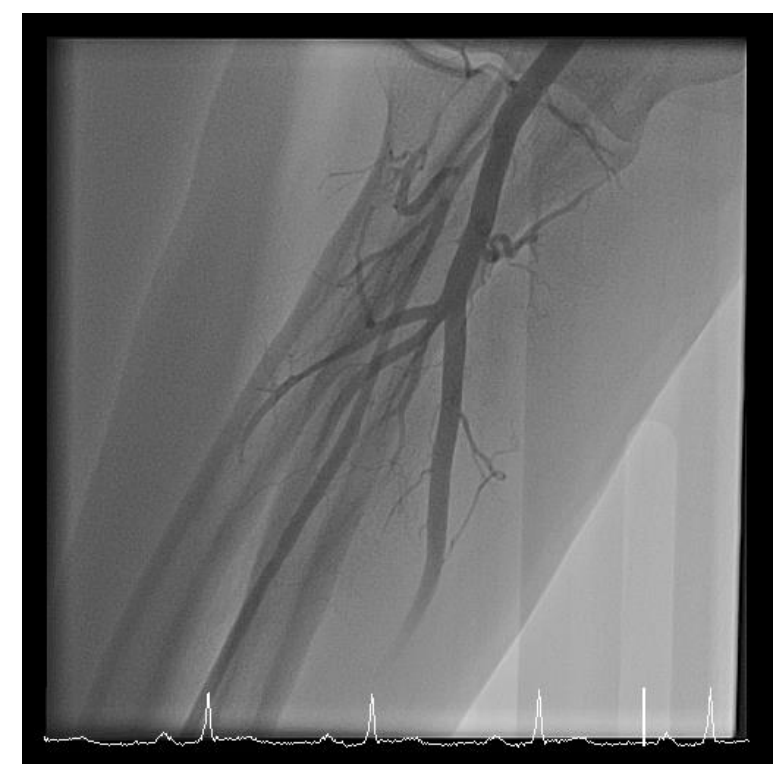

Figure $\mathbf{2 b}$ - Sealed perforation of the radial artery in Patient B after BAT

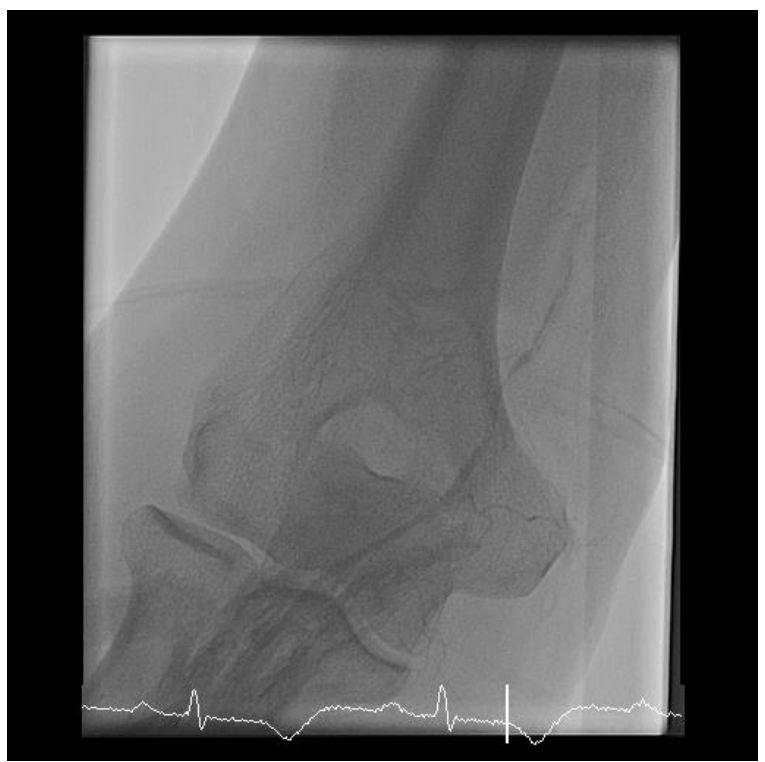

Figure 3a- Perforation of the radial artery in Patient C 


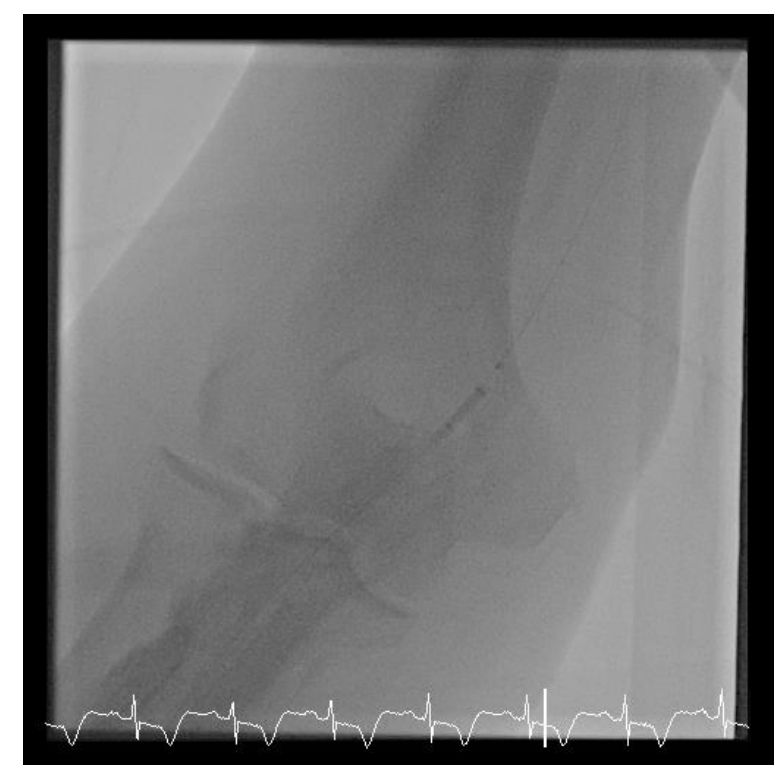

Figure $3 b-B A T$ of the guiding catheter in Patient $C$

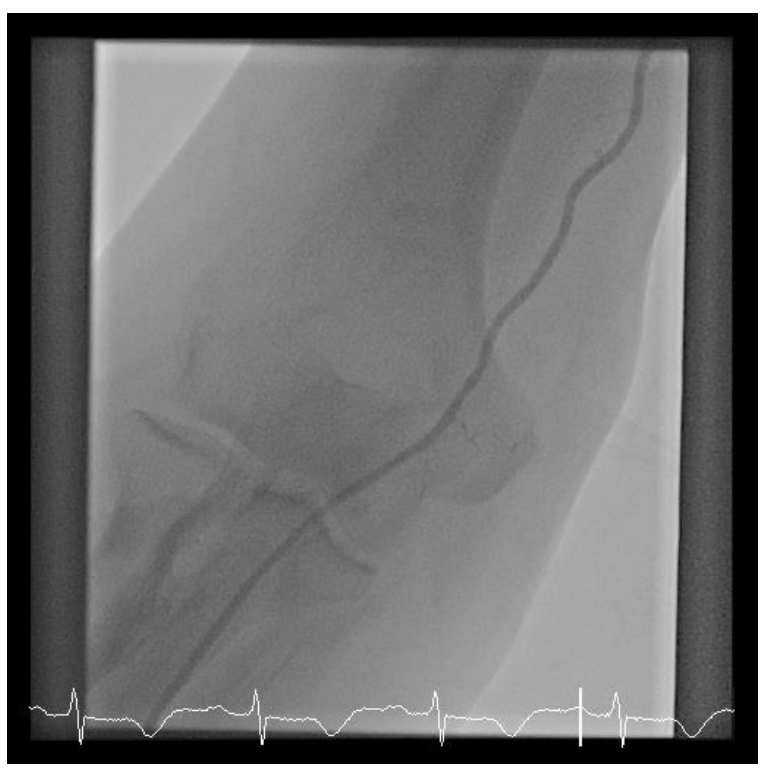

Figure 3c - Sealed perforation of the radial artery in Patient C after BAT 


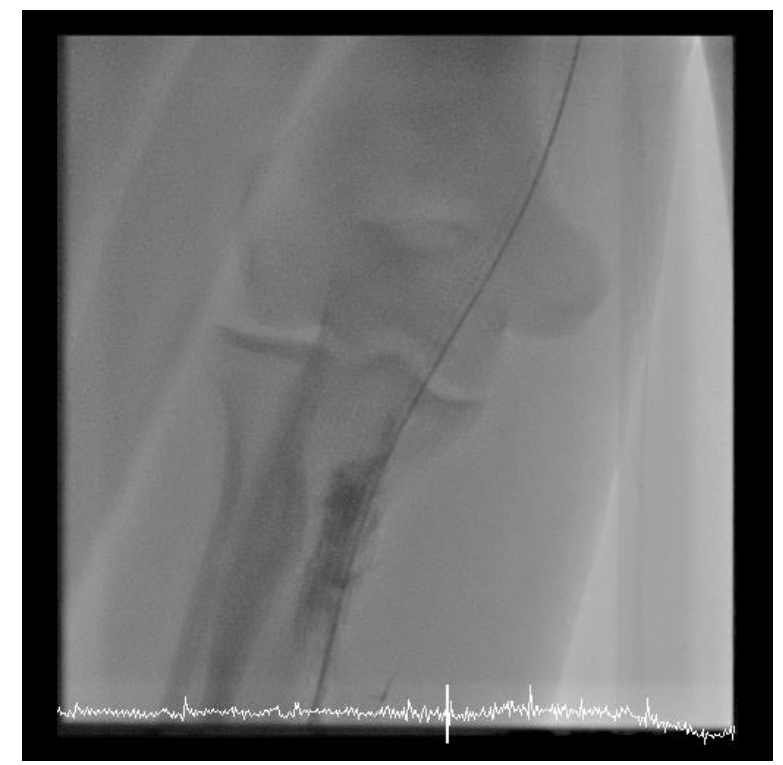

Figure 4a- Perforation of the radial artery in Patient $D$

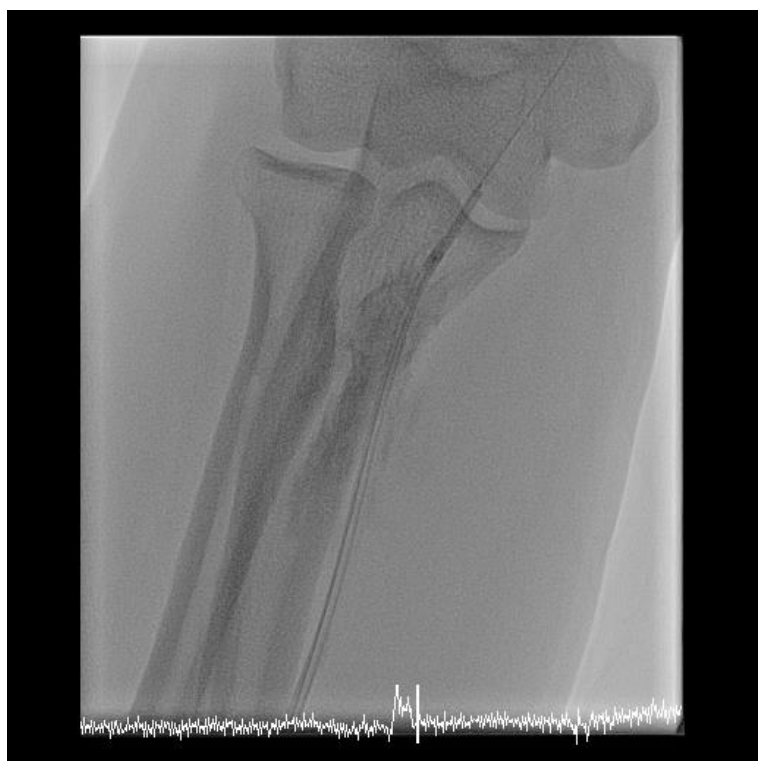

Figure 4b - BAT of the guiding catheter in Patient D 


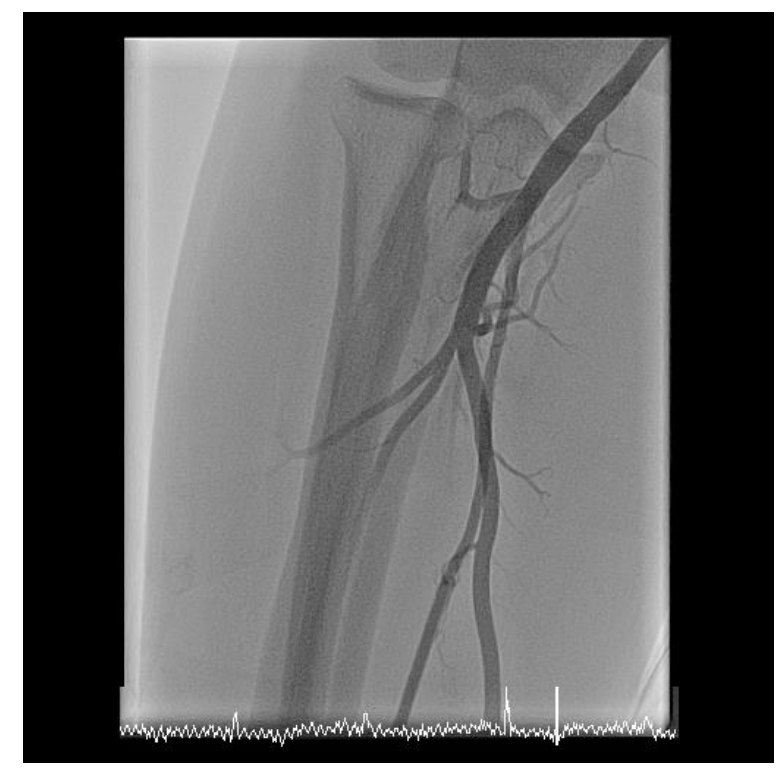

Figure $4 c$ - Sealed perforation of the radial artery in Patient $D$ after BAT

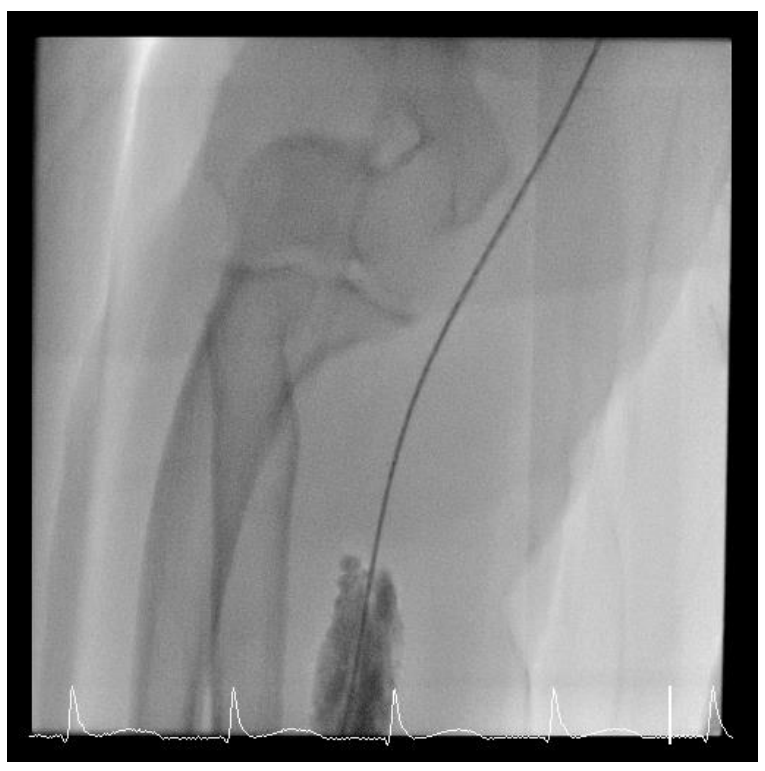

Figure 5a-Radial perforation in patient $E$ 


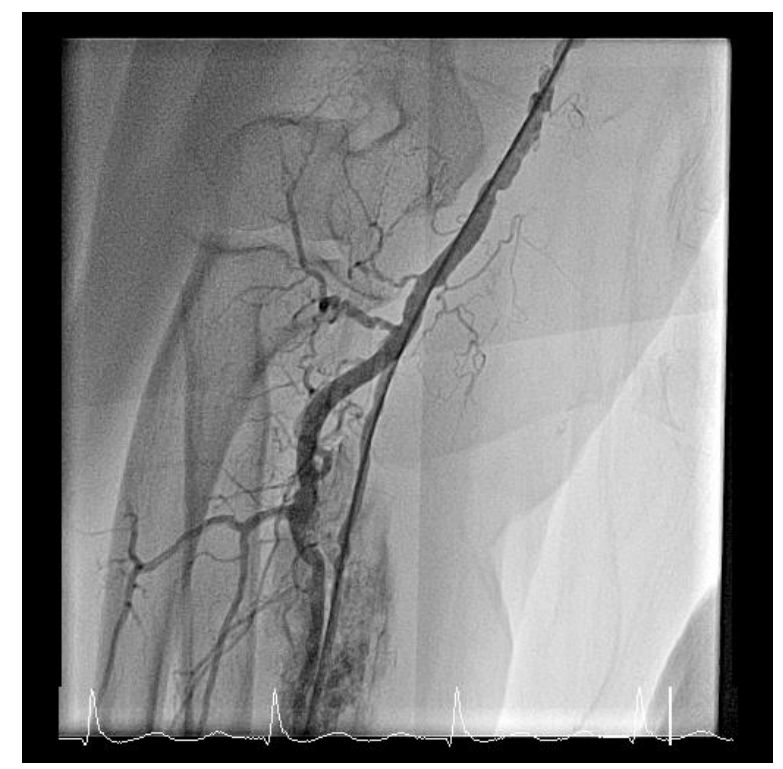

Figure $5 b$ - Radial artery open at end of procedure but note continued extravasation of contrast in patient $E$ confirming that perforation not sealed

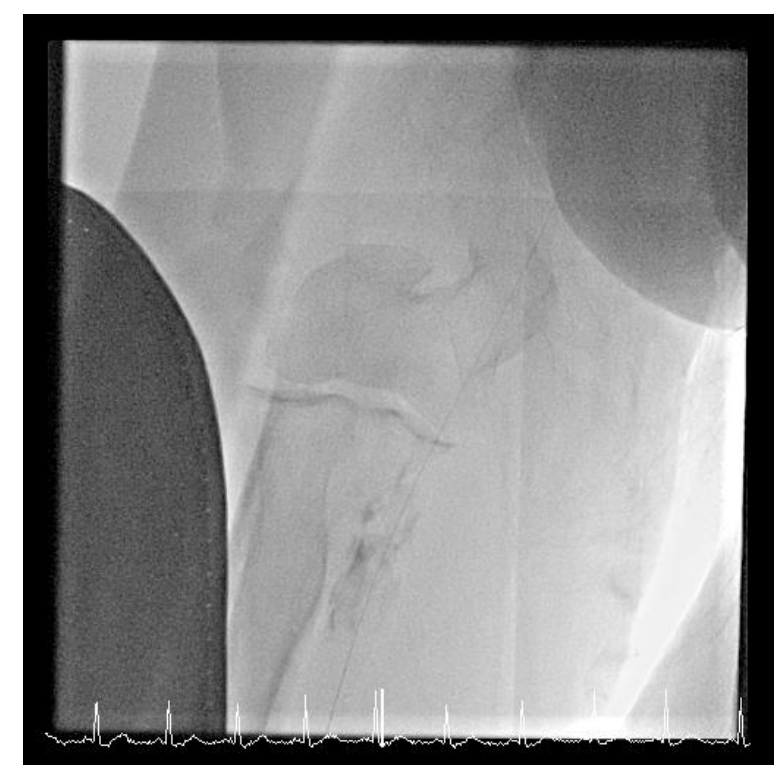

Figure 6a-Radial artery perforation in patient $\mathrm{F}$ 


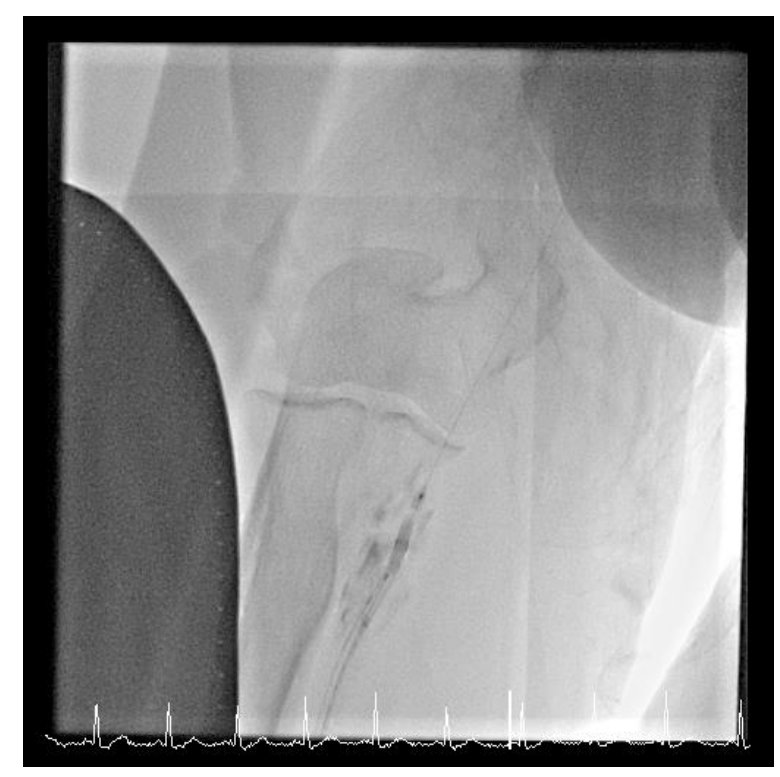

Figure $6 b-B A T$ in patient $F$

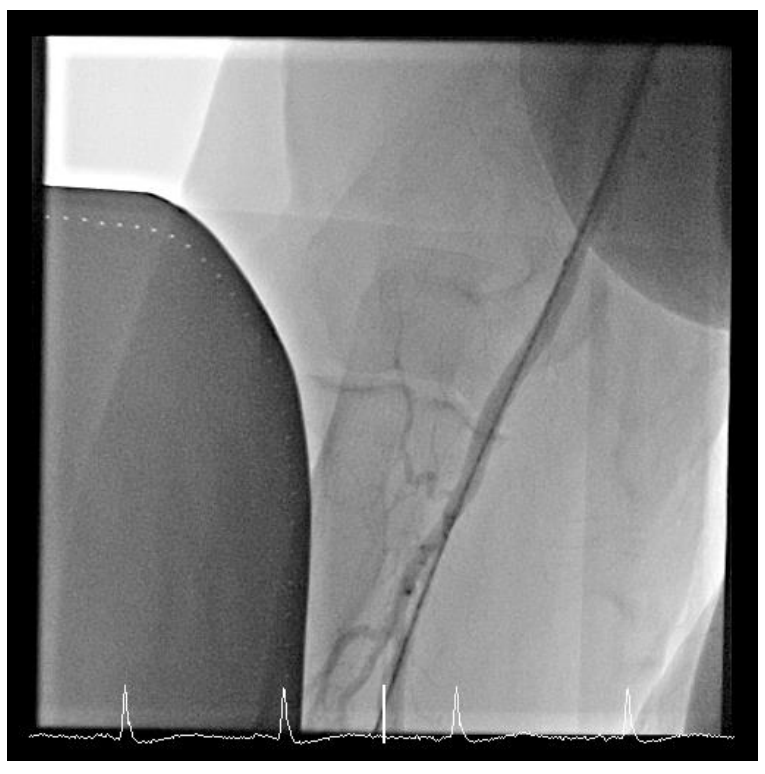

Figure $6 c-$ Sealed radial perforation in patient $F$ 


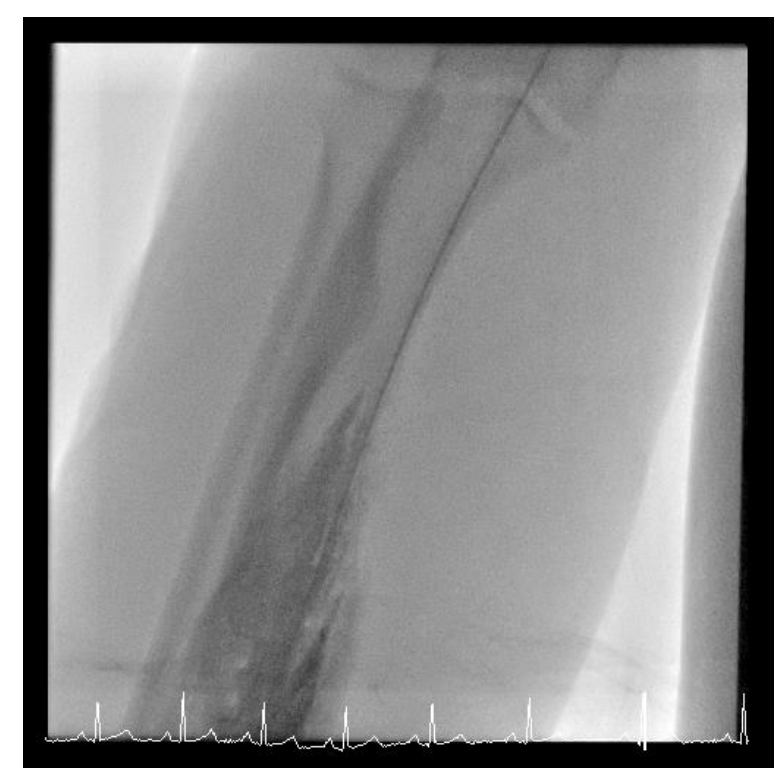

Figure 7a-Radial artery perforation in patient G

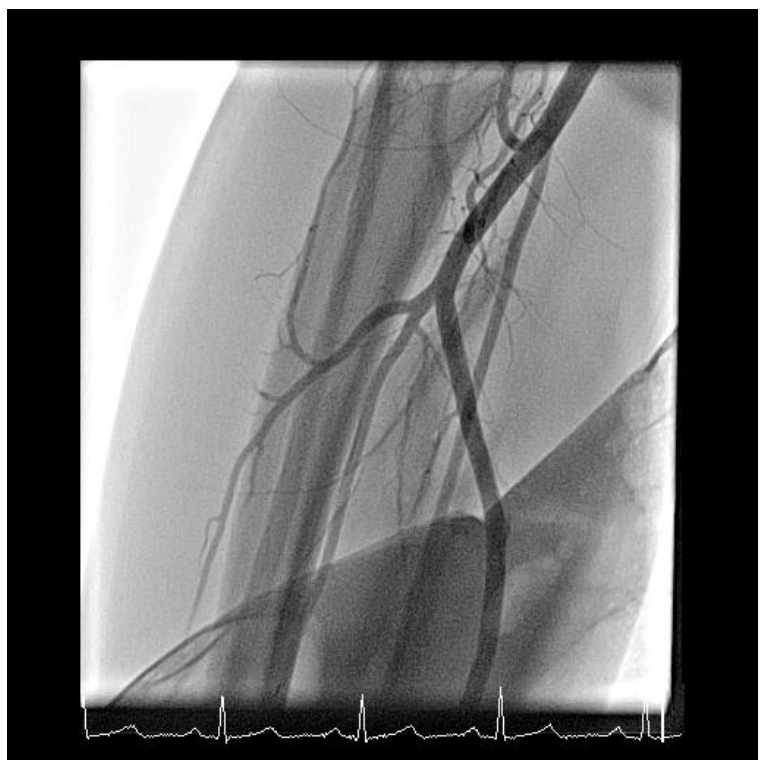

Figure $7 \mathrm{~b}$ - Sealed radial artery perforation after BAT in patient $\mathbf{G}$ 